\title{
A Prospective Randomized Controlled Study of the Role of Heparin in Preventing Deep Venous Thrombosis in Postoperative Craniotomy Patients
}

\author{
Navneet Singla, Durga Prasad Bendapudi, Rajesh Chhabra, Pankaj Malhotra, Mahesh Prakash, Sunil Kumar Gupta
}

\begin{abstract}
Background: Patients undergoing surgery for intracranial tumors are at significant risk of developing deep venous thrombosis (DVT) due to postoperative immobilization. The role of heparin prophylaxis in preventing this complication and the risk of intracranial hemorrhagic complications due to anticoagulation is not well established. The present study was designed to address this issue.
\end{abstract}

Materials and methods: A prospective randomized study was conducted to include 100 patients who were undergoing surgery for intracranial tumors. Patients who were immobilized in bed for more than 48 hours after surgery were included. The patients were categorized into two groups depending upon whether they received heparin prophylaxis or not. Further each group was subdivided into two based upon the duration of surgery: Less than or more than 3 hours. The incidence of DVT and of postoperative hemorrhagic complications was studied in all the groups. Various demographic as well as risk factors were analyzed to assess their impact on the incidence of DVT.

Results: Overall incidence of DVT was $17 \%$. Incidence of DVT was $12 \%$ in patients who received heparin as compared to $22 \%$ who did not. However, this difference did not reach statistical significance. The incidence was almost double when the duration of surgery was more than 3 hours. Patients with 2 or more risk factors had an increased frequency of DVT. One patient in the heparin positive group died as a result of postoperative hemorrhagic complication.

Conclusion: The incidence of DVT was found to be numerically lower in patients who received postoperative heparin as well as in patients in whom the duration of surgery was less than 3 hours.

Keywords: Deep venous thrombosis, Craniotomy, Heparin.

How to cite this article: Singla N, Bendapudi DP, Chhabra R, Malhotra P, Prakash M, Gupta SK. A Prospective Randomized Controlled Study of the Role of Heparin in Preventing Deep Venous Thrombosis in Postoperative Craniotomy Patients. J Postgrad Med Edu Res 2013;47(3):131-137.

Source of support: Nil

Conflict of interest: None declared

\section{INTRODUCTION}

Neurosurgical patients undergoing craniotomy for various reasons are at risk of deep venous thrombosis (DVT). ${ }^{1}$ According to many studies, incidence of DVT in patients undergoing craniotomy varies from 10 to $40 \%$ and the incidence of pulmonary embolism approaches $5 \% .{ }^{1,2}$ There are many risk factors that promote DVT and among them immobility is the most important risk factor for venous thromboembolism (VTE). ${ }^{3,4}$ Immobility can be due to primary disease per se or the postoperative state either due to the state of consciousness or due to the motor weakness. The risk is especially high in those having resection of brain tumors, especially malignant tumors. ${ }^{2,5}$ Prophylaxis with anticoagulant agents in neurosurgical patients has not gained wide acceptance because of concern about intracranial bleeding. On the other end of the spectrum is the risk of pulmonary embolism in neurosurgical patients which has been reported to be as high as $5 \%$, with a mortality rate ranging from 9 to $50 \%$. Heparin is widely used in the prophylaxis of VTE in moderateand high-risk clinical settings and it has been proven beyond doubt that prophylaxis in any form, i.e. mechanical or pharmacological, either alone or in combination decreases the incidence of DVT in postoperative patients. However, it is scarcely used in neurosurgical patients after routine intracranial surgery because of the potential consequences of bleeding in the brain or spine. This has created lot of confusion among all the neurosurgeons. The issue is whether or not the risk of bleeding can be minimized to warrant widespread use of anticoagulation instead of, or in addition to, mechanical prophylaxis in patients undergoing brain tumor surgery. To change the current standard of care new recommendations must be supported by trials in which both efficacy and safety have been studied, unfortunately few such studies exist and they do have limitations.

In this context our study aims to find the incidence of VTE in neurosurgical patients undergoing craniotomy, the risk factors that promote VTE and to study the complications of the heparin prophylaxis.

\section{MATERIALS AND METHODS}

A prospective randomized control study was performed. Hundred postoperative craniotomy patients operated between July 2009 and December 2010 in the Neurosurgery Department who meet the inclusion criteria were included in the study after randomization. 


\section{Inclusion Criteria}

- Patients who underwent craniotomy for intracranial mass lesions like meningiomas, gliomas, ependymomas, epidural or dural or intraventricular lesions.

- Patients who were bedridden for more than 48 hours postoperatively.

- Patients willing to participate in the study before the surgery for the primary pathology.

\section{Exclusion Criteria}

All the patients operated for:

- Trauma, spontaneous SAH or any infective lesions.

- Patients already under heparin prophylaxis preoperatively for various reasons.

- Patients with previous history of DVT or diagnosed case of DVT preoperatively.

- Patients with paralysis in the pre- or postoperative period.

- Patients who were ambulatory within 48 hours of the surgery.

- Patients with tumor bed hematoma postoperatively.

- Patients not willing to participate in the study.

All the patients who underwent craniotomy for the intracranial lesions enumerated in the inclusion criteria, excluding the patients in exclusion criteria were included in the study. This included the patients mostly operated for meningiomas, gliomas and other intracranial mass lesions. They underwent a qualitative preoperative serum D-dimer and compression ultrasound of the lower limbs to rule out the presence of DVT. The patients with positive or negative D-dimer were included in the study, but patients with positive compression ultrasound were excluded from the study. During the course of the hospital stay a thorough history regarding the factors which would lead to DVT were noted. The progress of the patient both clinically and sonographically was noted. All the relevant blood investigations were done and the tissue diagnosis obtained for each patient. All the patients underwent a CT scan within 24 hours and those with a significant intracerebral tumor bed hematoma were excluded from the study. The remaining patients who were bedridden for more than 48 hours were randomized according to the duration of the surgery (time from induction to reversal) into two groups. The randomization was done by card method. Group A: the duration of surgery was less than 3 hours. Group B: the duration of the surgery was more than 3 hours. Patients were randomized again into two groups. Groups A1 and B1 received heparin as prophylaxis and groups A2 and B2 did not receive heparin prophylaxis.
All the patients in the study group underwent a preoperative clinical examination (swelling, pain, Homan's sign), qualitative serum D-dimer and compression ultrasound of the lower limbs.

In the postoperative period all the patients were followed by clinical examination everyday, compression ultrasound of the lower limbs on postoperative day (POD) 3, POD 7, POD 10. All patients in groups A1 and B1 received either heparin (5,000 IU SC) thrice daily for 10 days or till the day of ambulation whichever is earlier. We categorized the patients based on the outcome in all the groups and the incidence of DVT was calculated in all the four groups, i.e. A1, B1, A2, B2. Those who developed DVT were categorized as: A1+, B1+, A2+, B2+. Those who did not develop DVT were categorized as: A1-, B1-, A2-, B2-. All the patients in whom DVT was not developed were followed up for 3 months for the development of DVT by clinical examination and by compression ultrasound of the lower limbs if clinically indicated. Patients who developed DVT in the postoperative period were treated according to the department protocol regardless of the group they were initially randomized to. All the patients underwent a repeat CT scan before discharge or in the course of follow-up as clinically indicated to evaluate the incidence of intracranial hemorrhage (ICH) and to compare the incidence between the heparinized and the nonheparinized group.

\section{Statistical Analysis}

Data was initially fed into Microsoft Excel worksheet to prepare the master chart and analyzed using statistical package for social science (SPSS version 19) software. The frequencies were calculated for the descriptive variable and its significance to the incidence of DVT was calculated by formulating the p-value with a Pearson Chi-square test. For the continuous variables, mean and standard deviation was calculated and its significance to the incidence of DVT was calculated by formulating the p-value with a MannWhitney's test. p-value $<0.05$ was considered to be significant and p-value $<0.001$ was considered to be highly significant.

\section{RESULTS (TABLE 1)}

\section{Incidence of DVT}

We studied a total of 100 patients of which 17 patients in total developed DVT.

The differential incidence was calculated (Table 2).

- A1 (24) <3 hours, heparin not given: 5 out of 24 (20.83\%) developed DVT.

- A2 (26) <3 hours, heparin given: 2 out of 26 (7.69\%) developed DVT. 


\begin{tabular}{|c|c|c|c|c|c|}
\hline \multicolumn{6}{|c|}{ Table 1: Overall data } \\
\hline \multicolumn{3}{|l|}{$D V T$} & Positive & Negative & $p$-value \\
\hline \multicolumn{3}{|l|}{ Total number } & 17 & 83 & \\
\hline \multicolumn{3}{|l|}{ Age (mean) } & 43.59 years & 39.81 years & 0.328 \\
\hline \multirow{2}{*}{ Sex } & \multicolumn{2}{|l|}{ Males } & 12 & 47 & 0.286 \\
\hline & \multicolumn{2}{|l|}{ Females } & 5 & 36 & \\
\hline \multirow{2}{*}{\multicolumn{3}{|c|}{$\begin{array}{l}\text { BMI } \\
\text { Number of days } \\
\text { of immobilization }\end{array}$}} & $27.78 \mathrm{~kg} / \mathrm{m}^{2}$ & $25.18 \mathrm{~kg} / \mathrm{m}^{2}$ & 0.089 \\
\hline & & & 11.94 days & 6.12 days & 0.000 \\
\hline \multirow[t]{15}{*}{ Risk factors } & \multicolumn{2}{|c|}{ History of hospitalization } & 8.88 days & 5.18 days & 0.249 \\
\hline & \multirow[t]{2}{*}{ Obesity } & Yes & 11 & 38 & 0.155 \\
\hline & & No & 6 & 45 & \\
\hline & \multirow[t]{2}{*}{ Smoking } & Yes & 6 & 18 & 0.231 \\
\hline & & No & 11 & 65 & \\
\hline & \multirow[t]{2}{*}{ Alcohol } & Yes & 7 & 13 & 0.017 \\
\hline & & No & 10 & 70 & \\
\hline & \multirow{2}{*}{ Diabetes mellitus } & Yes & 0 & 4 & 1.000 \\
\hline & & No & 17 & 79 & \\
\hline & \multirow[t]{2}{*}{ Hypertension } & Yes & 4 & 7 & 0.089 \\
\hline & & No & 13 & 76 & \\
\hline & \multirow[t]{2}{*}{ CAD } & Yes & 1 & 0 & 0.170 \\
\hline & & No & 16 & 83 & \\
\hline & \multirow[t]{2}{*}{ COPD } & Yes & 0 & 3 & 1.000 \\
\hline & & No & 17 & 80 & \\
\hline \multirow[t]{8}{*}{ Groups } & \multicolumn{2}{|l|}{ A1 } & 5 & 19 & - \\
\hline & \multicolumn{2}{|l|}{$\mathrm{A} 2$} & 2 & 24 & \\
\hline & \multicolumn{2}{|l|}{ B1 } & 6 & 19 & \\
\hline & \multicolumn{2}{|l|}{$\mathrm{B} 2$} & 4 & 21 & \\
\hline & \multicolumn{2}{|l|}{ Heparin given } & 6 & 45 & 0.155 \\
\hline & Heparin not given & & 11 & 38 & \\
\hline & Duration $<3$ hours & & 7 & 43 & - \\
\hline & Duration $>3$ hours & & 10 & 40 & \\
\hline Diagnosis & Vascular malforme & & 0 & 2 & 1.000 \\
\hline & Astrocytoma & & 2 & 8 & 1.000 \\
\hline & Oligodendrogliom & & 1 & 10 & 0.684 \\
\hline & Glioblastoma mult & me & 5 & 14 & 0.306 \\
\hline & Medulloblastoma & & 0 & 5 & 0.585 \\
\hline & PNET & & 0 & 3 & 1.000 \\
\hline & Ependymoma & & 3 & 0 & 0.004 \\
\hline & Craniopharyngiom & & 1 & 3 & 1.000 \\
\hline & Meningioma & & 2 & 19 & 0.354 \\
\hline & Schwannoma & & 2 & 6 & 0.620 \\
\hline & Miscellaneous & & 1 & 13 & 0.453 \\
\hline & Benign & & 5 & 39 & 0.283 \\
\hline & Malignant & & 12 & 44 & \\
\hline D-dimer & & & 8 & 29 & 0.346 \\
\hline Bleeding complica & & & 3 & 0 & 0.242 \\
\hline Mortality & & & 2 & 0 & - \\
\hline
\end{tabular}

Table 2: Differential incidence of DVT in each group

\begin{tabular}{|c|c|c|c|c|c|c|}
\hline & & & \multicolumn{4}{|c|}{ Groups } \\
\hline & & & $\begin{array}{l}<3 \text { hours, } \\
\text { heparin not } \\
\text { given }(A 1) \\
(n=24)\end{array}$ & $\begin{array}{l}<3 \text { hours, } \\
\text { heparin } \\
\text { given }(A 2) \\
(n=26)\end{array}$ & $\begin{array}{l}>3 \text { hours, } \\
\text { heparin not } \\
\text { given (B1) } \\
(n=25)\end{array}$ & $\begin{array}{l}>3 \text { hours, } \\
\text { heparin } \\
\text { given (B2) } \\
(n=25)\end{array}$ \\
\hline \multirow[t]{2}{*}{ DVT } & No $(n=83)$ & Count (\%) & $19(79.2 \%)$ & $24(92.3 \%)$ & $19(76.0 \%)$ & $21(84.0 \%)$ \\
\hline & Yes $(n=17)$ & Count (\%) & $5(20.8 \%)$ & $2(7.7 \%)$ & $6(24.0 \%)$ & 4 (16.0\%) \\
\hline
\end{tabular}

- $\quad$ B1 (25) >3 hours, heparin not given: 6 out of 25 (24\%) developed DVT.

- B2 (25) >3 hours, heparin given: 4 out of 25 (16\%) developed DVT.

With an overall risk of $17 \%$, the risk of incidence in the heparin positive groups (A2 and B2) appears to be two times higher when the duration of surgery is more than 3 hours, but the incidence of DVT appears to be same in the heparin negative groups (A1 and B1) irrespective of the duration of the surgery.

The incidence of DVT is calculated in another two groups. Heparin negative (A1 and B1, n = 49); 11 out of 49 
patients developed DVT (22\%). Heparin positive (A2 and B2, $n=51$ ); six out of 51 patients developed DVT (12\%). Though it was statistically insignificant (p-value: 0.155 ) but the incidence of DVT in heparin positive group was almost half of that in heparin negative group.

In the heparin negative group out of 11 patients who developed DVT, four patients developed DVT on day 3, four developed on day 7 and the rest three developed on day 10 following surgery. In the heparin positive group out of six patients who developed DVT, one patient developed on day 7, two on day 10, one on day 16 and two developed on day 20 following surgery. So heparin prophylaxis appeared to delay the onset of DVT.

\section{Age-DVT}

The mean age was 40.45 years with a minimum of 7 years and a maximum of 68 years. The mean age in the negative group was 39.81 years and in the positive group was 43.59 years. Though the mean age in those who developed DVT was higher than in patients who did not develop DVT, this factor was insignificant (p-values: 0.328). Out of 17 patients who were positive for DVT, eight were less than 40 years and nine were more than 40 years of age. This factor was also insignificant (p-value: 1.000).

\section{Sex-DVT}

Out of 100 patients $41 \%$ were females and $59 \%$ were males. Five females (12.2\%) and 12 males (20.3\%) developed DVT. This finding was insignificant (p-value: 0.286 ).

\section{Diagnosis-DVT}

All the patients who underwent craniotomy for various reasons, their histological diagnosis is taken as the final diagnosis and its relation to the incidence of DVT was studied.

The p-value was significant only in the case of ependymoma and insignificant in others. Out of the three patients with ependymoma, all were males, two were adults and one was a child, two were in the heparin negative group and one was in the heparin positive group, one had duration of surgery less than 3 hours, two had more than 3 hours, and the mean days of immobilization postsurgery was 22 days.

\section{DVT-Benign vs Malignancy}

Out of 100 patients 44 had benign disease and 56 had malignant disease. Five out of 17 (29.4\%) who were positive for DVT had a benign disease and 12 out of 17 (70.6\%) who were positive for DVT had a malignant disease. This finding was insignificant with a p-value of 0.283 .

\section{BMI-DVT}

The mean BMI was $25.61 \mathrm{~kg} / \mathrm{m}^{2}$ with a minimum of $12.6 \mathrm{~kg} / \mathrm{m}^{2}$ and a maximum of $39.11 \mathrm{~kg} / \mathrm{m}^{2}$. The mean BMI in the DVT negative group was $25.18 \mathrm{~kg} / \mathrm{m}^{2}$ and in the DVT positive group were $27.78 \mathrm{~kg} / \mathrm{m}^{2}$. The above finding was insignificant with a p-value of 0.089 .

\section{No. of Days of Immobilization-DVT}

The mean duration of immobilization after craniotomy was 7.11 days with a minimum of 4 days and a maximum of 31 days. The mean duration of immobilization in the DVT negative group was 6.12 days and in the DVT positive group was 11.94 days. Mann-Whitney test was applied and this finding was found to be very highly significant ( $\mathrm{p}$-value $<0.001$ ) with a p-value of 0.000 .

\section{Risk Factors-DVT}

Of 100 patients 49 were obese (BMI more than 25), 11 out of 17 for DVT positive were obese and the remaining six were not obese. This finding was insignificant with a p-value of 0.155 . Out of 100 patients, 24 were smokers. Six patients among the 17 who had positive DVT were smokers and the remaining 11 patients were nonsmokers. Out of 100 patients studied, 20 patients were alcoholics. Out of the 17 patients seven were alcoholics and the remaining 10 were not. This finding was significant with a p-value of 0.017 . Out of 100 patients studied, four patients were diabetic. Out of the 17 patients who were positive none were diabetic. This finding was insignificant with a p-value of 1.000 . Out of 100 patients studied, 11 patients were hypertensive. Out of the 17 patients who were positive four were hypertensive and the remaining 13 were not. This finding was not significant with a p-value of 0.089 . Out of 100 patients studied, one patient had a history of CAD. Out of the 17 patients who were positive one patient had a history of CAD. This finding was insignificant with a p-value of 0.170 . Out of 100 patients studied, three patients had history of COPD. Out of the 17 patients who were positive none had COPD. This finding was insignificant with a p-value of 1.000 .

Incidence of DVT in relation to multiple risk factors: The incidence of DVT was found to be significant when the patient had more than one risk factor. Out of the 17 patients who were positive for DVT three patients had no risk factors, five patients had 1 risk factor, four patients had 2 risk factors, four patients had 3 risk factors and one patient had 4 risk factors. Thirty-four patients did not have 
any risk factors and out of these three were positive for DVT and remaining 31 were negative. Thirty-seven patients had 1 risk factor out of which five were positive and 32 were negative for DVT. Seventeen patients had 2 risk factors out of which four were positive and 13 were negative for DVT. Seven patients had 3 risk factors out of which four were positive and three were negative for DVT. Five patients had 4 risk factors out of which one was positive and four were negative for DVT. This finding was significant with a p-value of 0.033 .

\section{D-Dimer-DVT}

Out of 100 patients studied, 37 patients had a positive preoperative D-dimer. Out of the 17 patients who were DVT positive eight had a positive $\mathrm{D}$-dimer test and the remaining nine were negative for the $\mathrm{D}$-dimer. This finding was insignificant with a p-value of 0.346 .

\section{Side of DVT}

Three patients had DVT in both the limbs. Two patients had left-sided DVT whereas 16 patients had right-sided DVT.

\section{Follow-up and Bleeding Complications}

None of the patients out of 50 had ICH during follow-up in those not given heparin, but three out of 50 patients developed ICH during follow-up in those given heparin. This finding was insignificant with a p-value of 0.242 . Out of the 17 patients who developed DVT, in 14 patients DVT disappeared on follow-up compression ultrasound, in two patients it first progressed to involve the distal veins and then regressed and one patient died. None of the patients had signs and symptoms of pulmonary embolism. The mortality rate in our study was $2 \%$ (2 out of 100 ). One patient was a case of ependymoma who died on postoperative day 10. The patient also had DVT but no ICH during the hospital stay. The death was related to the primary intracranial pathology. Other patient was a case of recurrent glioblastoma multiforme who died on postoperative day 4 . This patient had an intracranial hemorrhage on postoperative day 3. On the CT scan there was a significant tumor bed hematoma. This patient was on the prophylactic dose of heparin in the postoperative period. This patient also had DVT on postoperative day 3 and he died on the fourth postoperative day due to elevated intracranial pressure.

\section{DISCUSSION}

DVT is most commonly seen after immobilization and less commonly in some hematological disorders where there is imbalance of the coagulation cascade. Postoperative state is one such state which is known to predispose to DVT. ${ }^{4}$ This may be due to postoperative immobilization or due to the coagulation abnormalities like increase of the procoagulant factors and decrease of the anticoagulant factors due to surgery. There are studies indicating that DVT can occur silently without any symptoms and can progress to pulmonary thromboembolism which would be fatal. ${ }^{1}$ So the DVT and PE can add to the mortality and morbidity of the primary disease significantly. Patients undergo risk stratification and those falling into moderate- and high-risk groups may receive prophylaxis. The prophylaxis can be either mechanical or pharmacological or both. It had been proven beyond doubt that the prophylaxis started in the preoperative period had significantly decreased the incidence of DVT especially in the high-risk groups. ${ }^{6} \mathrm{~A}$ meta-analysis that examined all trials using heparins noted a relative risk reduction of $45 \%$ in thrombosis; the number needed to treat to prevent one VTE was 7.7. There was a 2-fold increase in bleeding rates, but major bleeding events did not differ between heparin-treated and control groups. ${ }^{7}$ Though mechanical prophylaxis had few side effects, pharmacological prophylaxis had its own adverse effects like increased chances of bleeding and thrombocytopenia which also would add to the mortality and morbidity of the primary disease. ${ }^{8}$ There are many studies to indicate pharmacological prophylaxis either alone or in combination with the mechanical prophylaxis which had a better outcome over the mechanical prophylaxis alone. In the study by Cerrato et al $4 \%$ of patients who were given subcutaneous UH beginning 2 hours preoperatively and every 8 hours thereafter developed a hematoma vs $2 \%$ of those in the control group. ${ }^{1}$ These factors have led to a state of confusion among all the neurosurgeons whether or not to use prophylaxis for preventing DVT.

Even after extensive studies on VTE and various modalities in preventing it, whether or not use anticoagulants, if at all to be used, when should they be started and until what time should they be continued, what dose to be used, who are the subset of beneficiaries, especially in the present day neurosurgical practice remains unanswered completely.

The overall incidence of DVT in our study was $17 \%$. Patients in the all the four groups developed DVT, i.e. in patients with duration of surgery less than and more than 3 hours and in patients receiving and not receiving heparin. The differential incidence of DVT in all the four groups, i.e. group A1 was $20.83 \%$, group A2 was $7.69 \%$, group $\mathrm{B} 1$ was $24 \%$ and in group B2 was $16 \%$. The differential 
incidence of DVT in the heparin positive and negative group was 12 and $22 \%$ respectively. Although, numerically it appears that the incidence in the negative group was double that of the positive group, it was found to be statistically insignificant. The risk of incidence in the heparin positive group appears to be two times higher when the duration of surgery is more than 3 hours, but the incidence of DVT appears to be same in the heparin negative group irrespective of the duration of the surgery. In our study, heparin prophylaxis delayed the onset of DVT.

Of the various parameters analyzed, the duration of immobilization in the postoperative period was found to be highly significant for the risk of developing DVT.

We considered many risk factors like obesity, history of previous hospitalization, smoking, alcohol, diabetes mellitus, hypertension, CAD and COPD that might promote DVT in our study. The risk factors already known to cause DVT like presence of coagulopathy, paralytic limbs were excluded from the study. Alcohol as a risk factor showed a significant relation to the incidence of DVT. Among the other risk factors, in obese and CAD patients the incidence of DVT was numerically higher but was statistically insignificant and the remaining risk factors like history of previous hospitalization, smoking, diabetes, hypertension, COPD had a numerically lower incidence and is also statistically insignificant. We divided the patients according to the number of the risk factors and its influence in the incidence of DVT was calculated. We found that patients having 2 or more than 2 risk factors showed a statistically significant increase in the incidence of DVT. This finding is supported in many studies that when the patients being smoker or alcoholic or obese when immobilized for a long duration had a higher chance of developing DVT. ${ }^{9-11}$ Literature supports the finding that the incidence of DVT is directly proportional to the duration of postoperative immobilization. ${ }^{4}$ Most of the other risk factors were not evaluated and maybe further studies are required for the other possible risk factors. As our study design was small, a larger study is required to elicit the final conclusion.

The total incidence of bleeding complications was seen in $3 \%$. All the patients who developed bleeding complications were in the heparin positive group. One patient died on postoperative day 4 and the other two patients were operated and the clot evacuated and heparin discontinued. Those patients who were operated recovered uneventfully. None of the patients in the heparin negative group developed ICH during follow-up. Though the number was higher compared to that reported in the literature; this finding was statistically insignificant. Out of the 17 patients, in 14 patients DVT regressed, in two patients it first progressed and then regressed and one patient died. None of the patients had signs and symptoms of pulmonary embolism.

\section{CONCLUSIONS AND LIMITATIONS OF THE STUDY}

The incidence of DVT was found to be numerically lower in patients who received postoperative heparin as well as in patients in whom the duration of surgery was more than 3 hours. The risk was also more in the presence of 2 or more associated risk factors. The main limitation of this study is its small size. To arrive at a logical conclusion as to whether routine use of heparin in postcraniotomy patients is beneficial in terms of risk reduction for DVT and whether the risk of developing postoperative bleeding complications is acceptable or not; a larger multicentric study would be required.

\section{REFERENCES}

1. Cerrato D, Ariano C, Fiacchino F. Deep vein thrombosis and low-dose heparin prophylaxis in neurosurgical patients. J Neurosurg 1978;49:378-81.

2. Constantini S, Kornowski R, Pomeranz S, et al. Thromboembolic phenomena in neurosurgical patients operated upon for primary and metastatic brain tumours. Acta Neurochir 1991;109:93-97.

3. Brandes AA, Scelzi E, Salmistraro G, Ermani M, Carollo C, Berti $\mathrm{F}$, et al. Incidence and risk of thromboembolism during treatment of high-grade gliomas: A prospective study. Eur J Cancer 1997;33:1592-96.

4. Macdonald RL, Amidei C, Lin G, et al. Safety of perioperative subcutaneous heparin for prophylaxis of venous thromboembolism in patients undergoing craniotomy. Neurosurgery 1999;45:245-52.

5. Sawaya R, Decourteen-Meyers G, Copeland B. Massive preoperative pulmonary embolism and suprasellar brain tumor: Case report and review of the literature. Neurosurgery 1984;15: 566-71.

6. Bostrom S, Holmgren E, Jonsson O, et al. Postoperative thromboembolism in neurosurgery. A study on the prophylactic effect of calf muscle stimulation plus dextran compared to lowdose heparin. Acta Neurochir 1986;80:83-89.

7. Iorio A, Agnelli G. Low-molecular-weight and unfractionated heparin for prevention of venous thromboembolism in neurosurgery: A meta-analysis. Arch Intern Med 2000;160: 2327-32.

8. Vanek VW. Meta-analysis of effectiveness of intermittent pneumatic compression devices with a comparison of thigh high to knee-high sleeves. Am Surg 1998;64:1050-58.

9. Chan AT, Atiemo A, Diran LK, et al. Venous thromboembolism occurs frequently in patients undergoing brain tumor surgery despite prophylaxis. J Thromb Thrombolysis 1999;8: 139-42. 
10. Levi AD, Wallace MC, Bernstein M, et al. Venous thromboembolism after brain tumor surgery: A retrospective review. Neurosurgery 1991;28:859-63.

11. Missori P, Lunardi P, Salvati M, et al. Pulmonary embolism in neurosurgical patients. Neurochirurgia 1991;34:170-73.

\section{ABOUT THE AUTHORS}

\section{Navneet Singla (Corresponding Author)}

Assistant Professor, Department of Neurosurgery, Postgraduate Institute of Medical Education and Research, Chandigarh, India e-mail: drnavi2007@yahoo.co.in

\section{Durga Prasad Bendapudi}

Junior Resident, Department of General Surgery, Postgraduate Institute of Medical Education and Research, Chandigarh, India

\section{Rajesh Chhabra}

Additional Professor, Department of Neurosurgery, Postgraduate Institute of Medical Education and Research, Chandigarh, India

\section{Pankaj Malhotra}

Additional Professor, Department of Internal Medicine, Postgraduate Institute of Medical Education and Research, Chandigarh, India

\section{Mahesh Prakash}

Associate Professor, Department of Radiodiagnosis and Imaging Postgraduate Institute of Medical Education and Research, Chandigarh India

\section{Sunil Kumar Gupta}

Professor, Department of Neurosurgery, Postgraduate Institute of Medical Education and Research, Chandigarh, India 